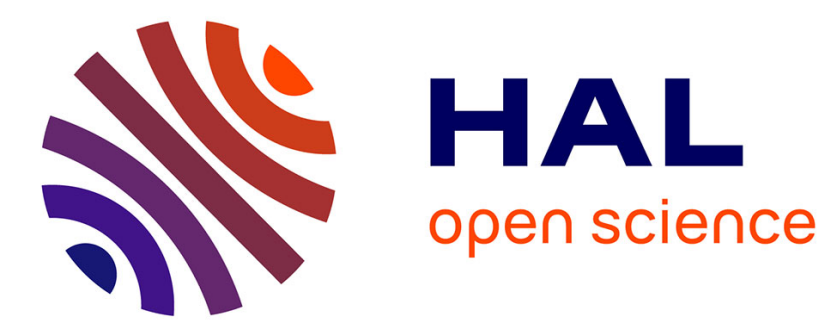

\title{
Reflection anisotropy spectra of polydimethylsiloxane under a range of mechanically applied stress
}

T Farrell, C I Smith, a L Schofield, R L Williams, P Weightman

\section{To cite this version:}

T Farrell, C I Smith, a L Schofield, R L Williams, P Weightman. Reflection anisotropy spectra of polydimethylsiloxane under a range of mechanically applied stress. Journal of Physics D: Applied Physics, 2010, 43 (24), pp.245301. 10.1088/0022-3727/43/24/245301 . hal-00569629

\section{HAL Id: hal-00569629 \\ https://hal.science/hal-00569629}

Submitted on 25 Feb 2011

HAL is a multi-disciplinary open access archive for the deposit and dissemination of scientific research documents, whether they are published or not. The documents may come from teaching and research institutions in France or abroad, or from public or private research centers.
L'archive ouverte pluridisciplinaire HAL, est destinée au dépôt et à la diffusion de documents scientifiques de niveau recherche, publiés ou non, émanant des établissements d'enseignement et de recherche français ou étrangers, des laboratoires publics ou privés. 


\title{
Reflection Anisotropy Spectra of Polydimethylsiloxane under a range of mechanically applied stress
}

\author{
T Farrell $^{1}$, C I Smith ${ }^{1}$, A L Schofield ${ }^{1}$, R L Williams ${ }^{2}$ and P Weightman ${ }^{1 *}$ \\ ${ }^{1}$ Department of Physics, University of Liverpool, Liverpool, L69 7ZE, UK \\ ${ }^{2}$ School of Clinical Sciences, University of Liverpool, L69 3GA, UK \\ *Corresponding Author
}

\begin{abstract}
Reflection anisotropy spectra (RAS) of transparent elastomeric polydimethylsiloxane (PDMS) have been determined over the spectral range $1.5 \mathrm{eV}$ to $5.0 \mathrm{eV}$ for a range of tensile loads. Using a slight wedge shaped sample the spectra from the incident air/PDMS surface are shown to be weak and largely featureless at all loads. By contrast the spectra from the light transmitted through the sample and reflected at the other interface are large in magnitude and exhibit features which arise from the small stress birefringence of PDMS.

The birefringent dominated spectra can readily be simulated using expressions derived from a Stokes-Mueller treatment of the optical system and this enabled the stress-optical coefficient to be determined at any energy within the spectral range covered. It is shown that there is a small angle between the optical axes and the mechanical axes in the sample and that this is consistent with the model of an elastomer which comprises a network of soft linear chains cross linked with stiff bridges.
\end{abstract}

\section{PACS}




\section{Introduction}

In the development of effective biomaterials and biomedical implants, the relationship between the cells and the surface of the material is important. Polymers such as polydimethylsiloxane (PDMS) should display good biocompatibility with the surrounding environment and it is cost effective and nontoxic [1]. PDMS displays high oxidative and thermal stability and can be fabricated into microstructures using soft lithography which makes it attractive for use in cell biology applications [2]. It is transparent over the visible and ultraviolet range down to $260 \mathrm{~nm}(4.7 \mathrm{eV})$ [3] and can be stretched to physiological linear extensions of up to $20 \%$, making it useful as stretchable culture substrates [1].

Mechanical forces can alter the organization and orientation of cells and proteins in tissues through a complex series of biochemical signals and mechanically induced changes. These are relevant to the development of biomaterials, as externally applied forces can be transmitted to adherent cells and regulate their behavior, for example through altered protein synthesis as takes place in tendons. Understanding how the biomaterial changes under conditions of mechanical strain is therefore crucial in the development of successful biomedical implants.

Reflection Anisotropy Spectroscopy (RAS) has the potential to provide information on the degree of anisotropy that takes place under stretching conditions. RAS was introduced in the 1980's as a monitor of semiconductor growth [4-6] and is now established as a powerful tool for the study of the surfaces of opaque centro-symmetric materials in which the bulk of the material is optically isotropic [7]. The technique has also been applied to study surfaces of birefringent materials [8].

The surfaces of many metals and semiconductors have been probed using RAS in ultra high vacuum (UHV) and Metal Organic Chemical Phase Deposition (MOCVD)/Metal Organic Vapour Phase Epitaxy (MOVPE) [7,9-12], and aqueous electrochemical environments [13-15]. In these materials the surface monolayer is very thin compared to the sampling depth and the wavelength of the probe light and on this basis theoretical models have been developed [16,17] which enable the RA spectra to be interpreted in terms of surface electronic transitions [17-19].

By comparison few RAS studies have been carried out on transparent dielectric materials $[8,20]$ although it has been long recognised that transparent windows used in UHV RAS studies may 
significantly affect the results. Indeed specific window designs are in use to reduce the effect of stress induced birefringence of the window material on the RA spectra.

As a preliminary to a planned RAS study of bio-molecules on relaxed and extended transparent PDMS we undertook a RAS investigation of PDMS over a range of mechanical strains. In this paper we report the results of those studies.

\section{Experimental}

The aim of these studies on PDMS was to provide a basis for RAS studies on bio-materials to be deposited on its surface [21]. Since PDMS is a transparent material the reflected light reaching the detector will not only come from the top surface (at the air/PDMS interface) there will also be a contribution from the light which has been transmitted through the sample and reflected at the lower interface. It is important that the RAS from the top surface is determined unambiguously; in other words it is desirable that any contribution arising from transmission and reflection at the lower surface is either eliminated or reduced to negligible proportions.

The RAS studies were carried out in a conventional phase modulated reflection anisotropy spectrometer [22], over the energy range $1.5 \mathrm{eV}$ to $5.0 \mathrm{eV}$. The angles of the analyser, photo elastic modulator (PEM) and polarizer are $0^{\circ}, 45^{\circ}$ and $-45^{\circ}$ with respect to the sample frame of reference. To facilitate the rotation of the sample under mechanical stress the spectrometer was mounted vertically.

The PDMS used in this study was Elastosil RT 601 A/B (Wacher Chemie AG, Germany). The PDMS was prepared from components A (the base polymer containing the catalyst) and B (the curing agent containing the cross linker) mixed in a 9:1 v/v ratio. The air was removed by degassing in a vacuum desiccator before the polymer was poured into petri dishes and left to set into sheet form, which took approximately 30 minutes at room temperature. Samples of the PDMS, nominally $1.0 \mathrm{~mm}$ thick, $2.0 \mathrm{~mm}$ wide and $10.0 \mathrm{~mm}$ gauge length, were pressed from the PDMS sheet into the classical dog-bone shape for use in the Tensile Stress Tester (Linkham Scientific Model TST350) which is capable of loads up to $200 \mathrm{~N}$. The tensile tester was mounted on a rotation stage which could be tilted about two orthogonal axes and translated in two orthogonal directions. After initial alignment to give the maximum total reflected signal the optical system was not adjusted. Prior to each spectrum the 
translation adjustments were used to ensure that the same area of the sample was being illuminated; also the maximum reflected signal was obtained prior by using the tilt adjustments, thus eliminating the influence of small changes in angle of incidence.

\section{Results}

Experiments were undertaken with PDMS samples pressed from sheets of uniform thickness and also from a sheet which was wedge shaped, the purpose of the latter being to separate the top and bottom reflections.

\subsection{Wedge Shaped Sample}

The angle of the wedge shaped sample was approximately $2^{\circ}$ in the plane normal to the applied load. With this sample two clearly defined beams typically $15.0 \mathrm{~mm}$ in diameter emerged and were visibly separate at the entrance port to the PEM, the separation approximately $20.0 \mathrm{~mm}$. As the stress was applied the sample distorted slightly as well as undergoing an extension and this resulted in a reduction of the separation of the beams at the larger extensions though they were still visibly separate to the eye. Spectra were taken over the range $1.5 \mathrm{eV}-5.0 \mathrm{eV}$ for the top surface and over the range $1.5 \mathrm{eV}-4.7 \mathrm{eV}$ for the bottom surface since above $4.7 \mathrm{eV}$ the dc signal fell dramatically due to absorption in the PDMS. Absorption in PDMS begins to rise at energies above $4.3 \mathrm{eV}$ [3].

The RAS results for the top surface of the wedge shaped sample are shown in figure 1 whilst those from the bottom surface are shown in figure 2. It is clear from figure 1 that extending the sample has little influence on the RA spectra, which remained weak and featureless for all extensions up to $10.0 \mathrm{~mm}$. At the higher extensions features appeared in the spectra but their magnitude was still very small. On the other hand spectra arising from the light which had been transmitted to the bottom surface and reflected exhibited very large changes as the stress level was increased. 

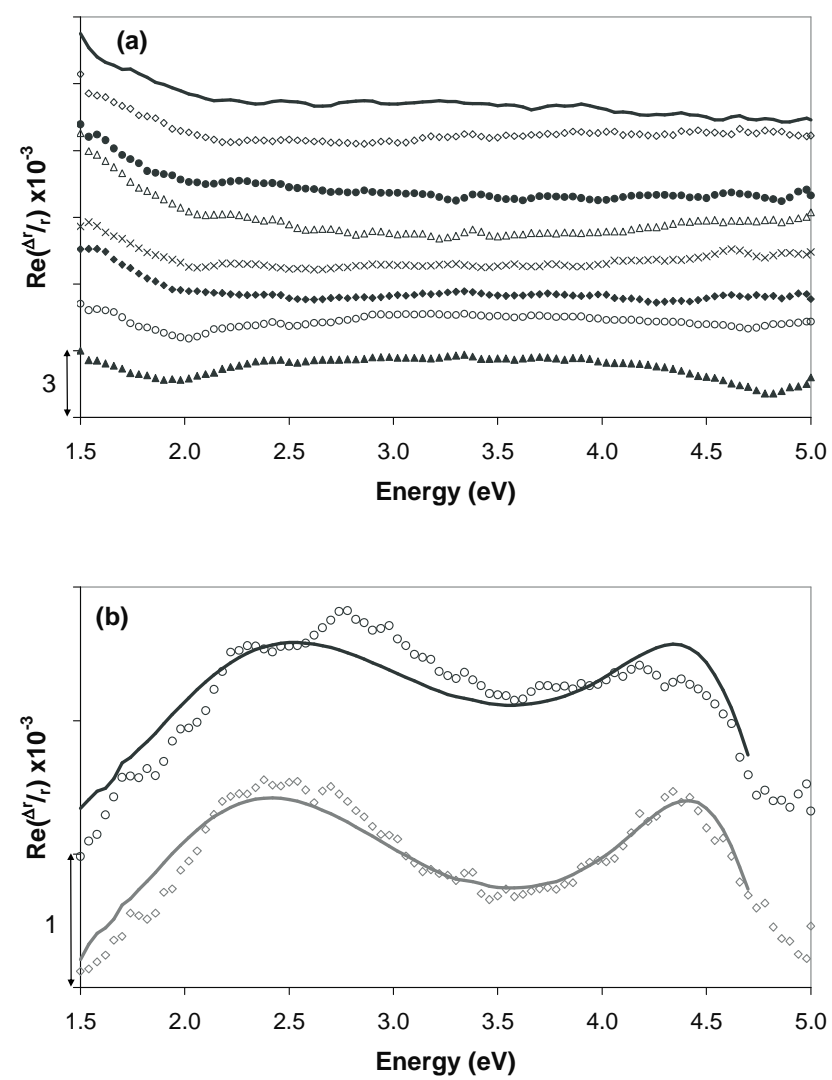

Figure 1. (a) RA Spectra from the upper surface of the wedge shaped sample of PDMS at $0.0 \mathrm{~mm}$ (solid line), $1.0 \mathrm{~mm}(\diamond), 2.0 \mathrm{~mm}(\bullet), 3.0 \mathrm{~mm}(\triangle), 4.5 \mathrm{~mm}(\times), 7.5 \mathrm{~mm}(\bullet), 10.0 \mathrm{~mm}(\bigcirc)$ and $12.5 \mathrm{~mm}(\mathbf{\Delta})$ extensions. The lines have been offset on the y-axis for clarity and the scale marked. (b) RA Spectra from the upper surface of the wedge shaped sample of PDMS at $15.0 \mathrm{~mm}(\square)$ and $16.0 \mathrm{~mm}(+)$ extensions. The solid lines are the scaled lineshapes obtained from the back surface as shown in figure 2.

If the spectra from the top and "bottom" reflections at the higher extensions are compared it is seen that the features in the spectra from the top surface occur at similar energies to the double maxima which occur in the "bottom" reflection. A least squares fitting procedure demonstrated that there was a very strong correlation between the features in the results from the upper surface and the form of the RA spectra from the lower surface. Thus in spite of the two beams still being visibly separate a small fraction of the bottom reflection is entering the PEM. In fact the least squares analysis shown in figure 1 for the $15.0 \mathrm{~mm}$ and $16.0 \mathrm{~mm}$ extension results indicated that this fraction was $(4.3 \pm 0.1) \times 10^{-3}$. 


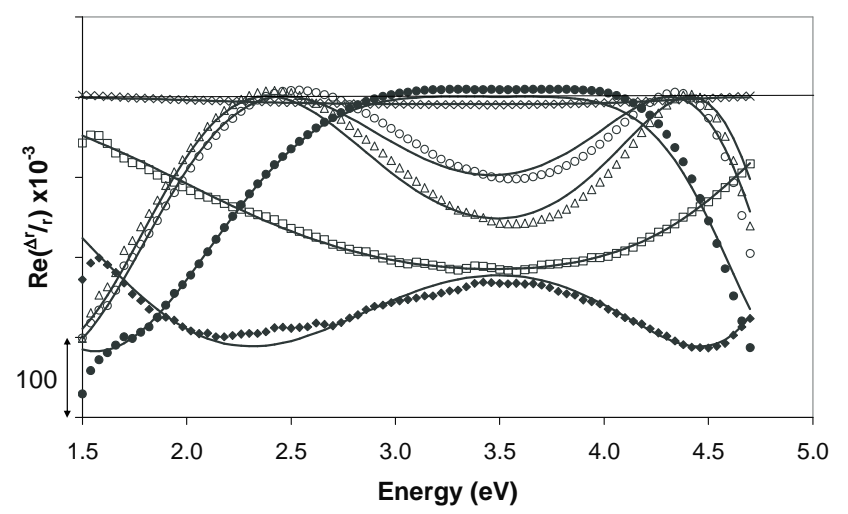

Figure 2. RA Spectra from the lower surface of the wedge shaped sample at $0.0 \mathrm{~mm}(\times), 3.0 \mathrm{~mm}(\square)$, $7.5 \mathrm{~mm}(\bullet), 12.5 \mathrm{~mm}(\bullet), 15.0 \mathrm{~mm}(\bigcirc)$ and $16.0 \mathrm{~mm}(\triangle)$ extensions with the corresponding simulations shown by the solid lines.

\subsection{Uniform thickness samples}

With samples of uniform thickness the reflection from the upper surface is not separated from that arising from the transmission and reflection from the lower surface and instead of a negligible or a very small contribution (c.f. $0.5 \%$ from the above wedge shaped results) close to $100 \%$ of the lower surface reflection will enter the PEM. The majority of the samples studied were of uniform thickness and the RA spectra obtained were intense and broadly similar to those shown in figure 2, indicating that the spectra result from light reflected from the lower surface. However the minimum sometimes occurred at a slightly different energy. In some samples the double minimum/maximum feature, which usually occurred at the larger extensions, was absent.

If now the lower surface of the sample is smeared with a film of viscous material having a similar refractive index to that of PDMS and such that there is no specular reflection from the viscous material/air interface then the large reflection from the lower surface of the PDMS should be reduced dramatically. Dow Corning high vacuum grease is a stable, silicone based translucent viscous material. This was smeared on to the lower surface of the PDMS sample and to further reduce the chances of reflection from this lower surface a matt-black strip was loosely attached to the grease film. Spectra from a uniform thickness sample are shown in figure 3. These spectra are featureless and 
weak from which it is evident that the contribution to RAS from the lower surface reflection is virtually totally suppressed.

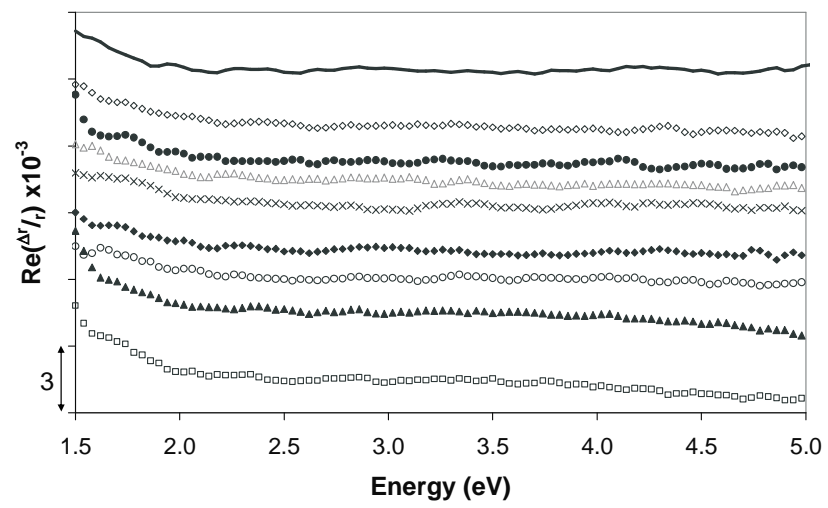

Figure 3. RA Spectra of a PDMS sample of uniform thickness with the lower surface reflection suppressed at $0.0 \mathrm{~mm}$ (solid line), $1.0 \mathrm{~mm}(\diamond), 2.0 \mathrm{~mm}(\bullet), 3.0 \mathrm{~mm}(\triangle), 5.0 \mathrm{~mm}(\times), 7.5 \mathrm{~mm}(\bullet)$, $10.0 \mathrm{~mm}(\bigcirc), 12.5 \mathrm{~mm}(\mathbf{\Delta})$ and $15.0 \mathrm{~mm}(\square)$ extensions. The lines have been offset on the y-axis for clarity and the scale marked.

\section{Theory}

A Stokes-Mueller treatment of the spectrometer indicates the parameters of the polarized light emerging from the sample. The Stokes vector of the light from the sample, assumed to be of unit intensity, is given by $S_{\mathrm{S}}$, where

$$
S_{S}=\left|\begin{array}{llll}
1 & q & u & v
\end{array}\right|^{T}
$$

In equation (1) the transpose of the vector is used to save space; the first term is the unit intensity, $q$ is the difference in intensity of two orthogonal linear polarisations, $u$ is the difference in intensity of two orthogonal polarisations but rotated by $\pi / 4$ from those relating to $q$ and $v$ is the difference in intensities of the left and right handed circular polarisations.

This polarized light passes through the PEM and the analyser to the monochromator and detector. The light entering the detector has a Stokes vector $S_{D}$ which is determined by Mueller calculus from 


$$
S_{D}=M_{A}^{0^{\circ}} M_{P E M}^{45^{\circ}} S_{S}
$$

where $M_{A}^{0^{\circ}}$ and $M_{P E M}^{45^{\circ}}$ are the Mueller matrices of the analyzer and PEM in the sample x/y Cartesian frame, see for example reference [23].

$$
M_{A}^{0^{\circ}}=\frac{1}{2}\left|\begin{array}{cccc}
1 & 1 & 0 & 0 \\
1 & 1 & 0 & 0 \\
0 & 0 & 0 & 0 \\
0 & 0 & 0 & 0
\end{array}\right| \quad M_{P E M}^{45^{\circ}}=\left|\begin{array}{cccc}
1 & 0 & 0 & 0 \\
0 & \cos \delta & 0 & -\sin \delta \\
0 & 0 & 1 & 0 \\
0 & \sin \delta & 0 & \cos \delta
\end{array}\right|
$$

Equation (2) then yields

$$
S_{D}=\left|\begin{array}{llll}
S_{0} & S_{1} & S_{2} & S_{3}
\end{array}\right|
$$

where $S_{0}=S_{1}=(1-v \sin \delta+q \cos \delta) ; S_{2}=S_{3}=0$.

The detector system records $S_{0}$ which comprises a time independent (DC) term of unit intensity and two time dependent (AC) terms via $\delta=\mathrm{A} \sin (2 \pi \mathrm{ft})$ where $f$ is the frequency of the PEM and A is the amplitude of the retardation. These AC terms can be expanded as Bessel Function series' [23] leading to

$$
S_{0} \approx V_{D C}+V_{1 f} \sin \delta+V_{2 f} \cos \delta
$$

Thus the $1 f$ term measures the difference between right handed and left handed circularly polarized light whilst the $2 f$ term measures the difference between the $\mathrm{x}$ and y components of linearly polarized light. The DC term measures the total intensity. It is usual in RAS studies at $2 f$ to set A to 3.054 radians since this is the argument at which the Bessel function $\mathrm{J}_{2}$ is maximum. However there is a contribution to the DC from the expansion of the term involving $2 f$ since $\mathrm{J}_{0}$ is non zero. The RAS results take this into account.

The full optical system can be analysed using Mueller calculus [23]. For this we need the Mueller matrices for reflection at the sample surfaces, transmission through the transparent sample and polarizer. Each must be rotated to be in the sample $x / y$ Cartesian frame, of which one of the principal axes was the direction of the applied tensile load. In our optical system the polarizer is at $-45^{\circ}$, the sample reflection is assumed to be at $0^{\circ}$ and for generality the transmission, which is regarded as being birefringent, is at $\alpha^{\circ}$. The matrices are 


$$
\begin{aligned}
M_{P}^{-45^{\circ}}=\frac{1}{2}\left|\begin{array}{cccc}
1 & 0 & -1 & 0 \\
0 & 0 & 0 & 0 \\
-1 & 0 & 1 & 0 \\
0 & 0 & 0 & 0
\end{array}\right| \\
M_{S R E F}^{0^{\circ}}=\left|\begin{array}{cccc}
\left(a^{2}+b^{2}\right)+\left(c^{2}+d^{2}\right) & \left(a^{2}+b^{2}\right)-\left(c^{2}+d^{2}\right) & 0 & 0 \\
\left(a^{2}+b^{2}\right)-\left(c^{2}+d^{2}\right) & \left(a^{2}+b^{2}\right)+\left(c^{2}+d^{2}\right) & 0 & 0 \\
0 & 0 & 2(a c+b d) & -2(a d-b c) \\
0 & 0 & 2(a d-b c) & 2((a c+b d)
\end{array}\right| \\
M_{S R E T}^{\alpha^{\circ}}=\left|\begin{array}{cccc}
1 \\
0 \\
0 & \cos ^{2}(2 \alpha)+\sin ^{2}(2 \alpha) \cos (\beta) & -\frac{1}{2} \sin (4 \alpha)(1-\cos (\beta)) & \sin (2 \alpha) \sin (\beta) \\
0 & -\frac{1}{2} \sin (4 \alpha)(1-\cos (\beta)) & \cos ^{2}(2 \alpha) \cos (\beta)+\sin ^{2}(2 \alpha) & \cos (2 \alpha) \sin (\beta) \\
0 & -\sin (2 \alpha) \sin (\beta) & -\cos (2 \alpha) \sin (\beta) & \cos (\beta)
\end{array}\right|
\end{aligned}
$$

In these matrices $a, b c$ and $d$ are defined from the amplitude reflection coefficients, $r_{x}$ and $r_{y}$ in the $\mathrm{x} / \mathrm{y}$ frame

$$
r_{x}=a-j b \quad r_{y}=c-j d
$$

whilst $\beta$ is the phase difference introduced by the retardation in the transparent sample.

We have used the general expression for the reflection coefficients; for transparent materials $b=d=0$. The intensity reflection coefficients are

$$
\mathrm{R}_{\mathrm{x}}=r_{x} r_{x}^{*}=\left(a^{2}+b^{2}\right) \quad \mathrm{R}_{\mathrm{y}}=r_{y} r_{y}^{*}=\left(c^{2}+d^{2}\right)
$$

The light from the xenon lamp is unpolarized and its Stokes vector, $S_{L}$ is

$$
S_{L}=\left|\begin{array}{llll}
1 & 0 & 0 & 0
\end{array}\right|^{T}
$$

The Stokes vector $S_{D}$ of the light incident on the monochromator/detector system having been reflected from the lower surface is calculated from

$$
S_{D}=M_{A}^{0^{\circ}} M_{P E M}^{45^{\circ}} M_{S R E T}^{\alpha^{\circ}} M_{S R E F}^{0^{\circ}} M_{S R E T}^{\alpha^{\circ}} M_{P}^{-45^{\circ}} S_{L}
$$

The evaluation of equation (7) leads to the column matrix (Stokes vector, c.f. equation (3))

$$
S_{D}=\left|\begin{array}{llll}
S_{0} & S_{1} & S_{2} & S_{3}
\end{array}\right|^{T}
$$

where $S_{0}=S_{1}$ and $S_{2}=S_{3} \equiv 0$.

$S_{0}$ measures the light entering the detector and is given by 


$$
\begin{aligned}
& 4 S_{0}=\left\lfloor\begin{array}{l}
\left\{\left(a^{2}+b^{2}\right)+\left(c^{2}+d^{2}\right)\right\} \\
-\frac{1}{2}\left\{\left(a^{2}+b^{2}\right)-\left(c^{2}+d^{2}\right)\right\} \sin (4 \alpha)(1-\cos (\beta))
\end{array}\right\rfloor \\
& +\left[\begin{array}{l}
2(a d-b c)\left\{\sin ^{2}(2 \alpha) \cos (\beta)+\cos ^{2}(2 \alpha) \cos (\beta)\right\} \\
-2(a c+b d) \cos (2 \alpha) \sin (\beta)\left\{\sin ^{2}(2 \alpha)+\cos ^{2}(2 \alpha) \cos (\beta)+\cos (\beta)\right\} \\
+\left\{\left(a^{2}+b^{2}\right)+\left(c^{2}+d^{2}\right)\right\} \sin (4 \alpha) \sin (2 \alpha) \sin (\beta)(\cos (\beta)-1) \\
-\left\{\left(a^{2}+b^{2}\right)-\left(c^{2}+d^{2}\right)\right\} \sin (2 \alpha) \sin (\beta)
\end{array}\right] \sin \delta \\
& +\left[\begin{array}{l}
\left\{\left(a^{2}+b^{2}\right)-\left(c^{2}+d^{2}\right)\right\}\left[\cos ^{2}(2 \alpha)+\sin ^{2}(2 \alpha) \cos (\beta)\right] \\
+\left\{\left(a^{2}+b^{2}\right)+\left(c^{2}+d^{2}\right)\right\} \frac{1}{2} \sin (4 \alpha)\left[\sin ^{2}(2 \alpha) \cos (\beta)+\cos ^{2}(2 \alpha)\right](1-\cos (\beta)) \\
+(a c+b d) \sin (4 \alpha)\left[\left(\sin ^{2}(2 \alpha)+\cos ^{2}(2 \alpha) \cos (\beta)\right)(\cos (\beta)-1)-\sin ^{2}(\beta)\right] \\
+2(a d-b c) \sin (2 \alpha)[\sin (2 \beta) \cos (2 \alpha)-\cos (4 \alpha) \sin (\beta)]
\end{array}\right]
\end{aligned}
$$

which, as above, is the sum of a DC term and two AC terms. When there is no birefringence $(\beta=0)$ the above reduces to

$$
S_{0}=0.25\left\{\left[\left(a^{2}+b^{2}\right)+\left(c^{2}+d^{2}\right)\right]+2(a d-b c) \sin \delta+\left[\left(a^{2}+b^{2}\right)-\left(c^{2}+d^{2}\right)\right] \cos \delta\right\}
$$

This is the result for the upper surface reflection, which for a transparent material is

$$
S_{0}=0.25\left\{\left(R_{x}+R_{y}\right)+\left(R_{x}-R_{y}\right)\right\} \cos \delta \approx 0.25(R+\Delta R \cos \delta)
$$

When $\alpha=0$ that is when the fast/slow optical axes coincide with the $\mathrm{x} / \mathrm{y}$ (mechanical) Cartesian frame of the sample, the expression becomes

$$
\begin{aligned}
S_{0}=0.25\{ & {\left[\left(a^{2}+b^{2}\right)+\left(c^{2}+d^{2}\right)\right]+[2(a d-b c) \cos (\beta)-(a c+b d) \sin (2 \beta)] \sin \delta } \\
+ & {\left.\left[\left(a^{2}+b^{2}\right)-\left(c^{2}+d^{2}\right)\right] \cos \delta\right\} }
\end{aligned}
$$

For a transparent material this reduces to

$$
S_{0} \approx R+\Delta R \cos \delta-a c \sin (2 \beta) \sin \delta
$$

It appears that the term $(a d-b c)$ which appears in equations (9) and (11) is associated with the handedness of the light reaching the detector. Its significance in RAS, when $\Delta r$ is small compared to $r$, can be seen by examining the ratio $2\left(r_{x}-r_{y}\right) /\left(r_{x}+r_{y}\right)=\Delta r / r$ where $r_{x}$ and $r_{y}$ and are given by equation (5). 


$$
\begin{aligned}
\frac{\Delta r}{r} & =2 \frac{(a-c)-j(b-d)}{(a+c)-j(b+d)} \times \frac{(a+c)+j(b+d)}{(a+c)+j(d+d)} \cong \frac{1}{2} \frac{\left(a^{2}+b^{2}\right)-\left(c^{2}+d^{2}\right)}{\left(a^{2}+b^{2}\right)+\left(c^{2}+d^{2}\right)}+j \frac{(a d-b c)}{\left(a^{2}+b^{2}\right)+\left(c^{2}+d^{2}\right)} \\
& =\operatorname{Re}\left(\frac{\Delta r}{r}\right)+j \operatorname{Im}\left(\frac{\Delta r}{r}\right)
\end{aligned}
$$

In this study we restricted our measurements to $\operatorname{Re}\left(\frac{\Delta r}{r}\right)$

\section{Discussion}

The first and practical aspect of the work is that the use of a wedge shaped sample to totally eliminate the unwanted lower surface reflected light is not entirely reliable. It is far better to use a reflection suppressing coating to achieve the desired result. However the wedge shaped sample has enabled an insight into the optical behaviour of PDMS, which we discuss below.

PDMS is generally regarded as having a low intrinsic birefringence and a low stress induced birefringence [24]. However figures for the stress-optical coefficient, K, vary widely. Values ranging from $13.5 \times 10^{-5}\left(\mathrm{MPa}^{-1}\right)$ at $20^{\circ} \mathrm{C}$ [24] and $632.8 \mathrm{~nm}$ to $1.7 \times 10^{-7}\left(\mathrm{MPa}^{-1}\right)$ at $633 \mathrm{~nm} \mathrm{[25]} \mathrm{are} \mathrm{quoted,}$ the lower value being attributed to slow curing of the PDMS.

If we assume that the RAS from the top surface is due to the difference in reflection arising from the stress birefringence then an indication of the magnitude of $\Delta R / R$ can be obtained from

$$
\frac{\Delta R}{R}=2\left(\frac{\left(\left(n_{1}-1\right) /\left(n_{1}+1\right)\right)^{2}-\left(\left(n_{2}-1\right) /\left(n_{2}+1\right)\right)^{2}}{\left(\left(n_{1}-1\right) /\left(n_{1}+1\right)\right)^{2}+\left(\left(n_{2}-1\right) /\left(n_{2}+1\right)\right)^{2}}\right)
$$

where $n_{1}$ and $n_{2}$ are the refractive indices in two orthogonal directions in the plane normal to the direction of incidence in the PDMS. The magnitude of $\Delta n=\left(n_{1}-n_{2}\right)$ can be estimated from the stress birefringence coefficient. Assuming $n_{1}$ is the refractive index of PDMS at $635 \mathrm{~nm}$ [26] and a birefringence coefficient of $13.5 \times 10^{-5}\left(\mathrm{MPa}^{-1}\right)$ then using equation (15) $\Delta R / R$ is in the range $0-5 \mathrm{x}$ $10^{-3}$ for the range of extensions in figure 3. In this estimation any dispersion in $n_{1}$ has been ignored; nonetheless the measured values of $\Delta R / R$ for the top surface are consistent with the above stress birefringence. 
It has recently been reported that $n_{1}$ is dispersive [27]. From the data and the Sellmeier fits [27] we can establish the variation of $n_{1}$ over the entire spectral range covered by our measurements. As PDMS is birefringent we can use $\Delta n$ as a fitting parameter and simulate the $\Delta R / R$ measurements using equation (9) and a least squares procedure. We assume in all cases that $n_{1}$ has the value calculated from reference [27]. We cannot assume that $\Delta n$ is a constant value over the entire spectral range and any dispersion is taken into account in the fitting procedure by allowing $\Delta n$ to exhibit a quadratic variation with photon energy, E.

$$
\Delta n=\mathrm{F}+\mathrm{GE}+\mathrm{HE}^{2}
$$

where $\mathrm{F}$ is the value of $\Delta n$ at $1.5 \mathrm{eV}$ - the lowest energy of the spectral range. Clearly $\Delta n$ estimated from equation (15) may not be monotonic but provided $n_{2}$ is monotonic and follows a Sellmeier formula then the empirical procedure is acceptable.

The phase difference $\beta$ varies with $\mathrm{E}$ according to

$$
\beta=\frac{4 \pi t \Delta n}{\lambda}=3.226 \pi t \Delta n E
$$

where $t$ is the sample thickness in $\mu \mathrm{m}$. In the unstressed condition the sample thickness was $1.1 \mathrm{~mm}$; clearly with increasing tensile stress the thickness reduces and this influences $\beta$. The thickness for each extension was estimated using the measured load and a Poisson's Ratio of 0.5 [28,29].

The fitting procedure therefore uses four parameters only: the angle $\alpha$ and the parameters $\mathrm{F}$, $\mathrm{G}$ and $\mathrm{H}$ which describe $\Delta n$. The simulated spectra are shown alongside the measured spectra in figure 2 from which it is seen that there is good agreement in all cases. The values of the fitting parameters are given in table 1 . There is some scatter in $\mathrm{F}, \mathrm{G}$ and $\mathrm{H}$ but the purpose of including them in the table is to enable the calculation of $\Delta n$ and hence the stress optical coefficient at any energy in the range covered by this work. In fact using $\Delta n$ obtained from $\mathrm{F}, \mathrm{G}$ and $\mathrm{H}$ leads to $n_{2}$ which is monotonic. In figure 4 we present the estimated values of $\Delta n$ as a function of engineering stress at three energies including $1.96 \mathrm{eV}(632.7 \mathrm{~nm}$, the HeNe laser wavelength). From the slope of a linear least squares fit, the stress optical coefficient is found to be $(7.7 \pm 0.2) \times 10^{-5} \mathrm{MPa}^{-1}$. This compares favourably with the value $13.5 \times 10^{-5} \mathrm{MPa}^{-1}$ given in reference [24]. 
Table 1. Values of the fitting parameters used in the spectra simulations.

\begin{tabular}{ccccc}
\hline Extension $(\mathrm{mm})$ & $\alpha$ degrees & $\mathrm{F}$ & $\mathrm{G}$ & $\mathrm{H}$ \\
\hline 0.0 & 0.062 & $6.60 \times 10^{-5}$ & $-7.444 \times 10^{-5}$ & $1.291 \times 10^{-5}$ \\
1.0 & 0.344 & $2.51 \times 10^{-5}$ & $-4.730 \times 10^{-5}$ & $8.54 \times 10^{-5}$ \\
2.0 & 0.859 & $5.70 \times 10^{-5}$ & $-3.450 \times 10^{-5}$ & $6.51 \times 10^{-5}$ \\
3.0 & 1.57 & $4.30 \times 10^{-5}$ & $-3.450 \times 10^{-5}$ & $6.51 \times 10^{-5}$ \\
4.5 & 1.89 & $-6.50 \times 10^{-5}$ & $1.190 \times 10^{-5}$ & $0.193 \times 10^{-5}$ \\
7.5 & 2.01 & $-1.00 \times 10^{-5}$ & $-6.20 \times 10^{-5}$ & $1.21 \times 10^{-5}$ \\
10.0 & 1.83 & $-4.50 \times 10^{-5}$ & $-7.495 \times 10^{-5}$ & $1.566 \times 10^{-5}$ \\
12.5 & 2.05 & $-9.00 \times 10^{-5}$ & $-9.60 \times 10^{-5}$ & $2.092 \times 10^{-5}$ \\
13.5 & 2.10 & $-1.30 \times 10^{-4}$ & $-1.01 \times 10^{-4}$ & $2.318 \times 10^{-5}$ \\
15.0 & 2.11 & $-1.505 \times 10^{-4}$ & $-1.051 \times 10^{-4}$ & $2.443 \times 10^{-5}$ \\
16.0 & 2.03 & $-1.42 \times 10^{-4}$ & $-1.502 \times 10^{-4}$ & $3.260 \times 10^{-5}$ \\
\hline
\end{tabular}

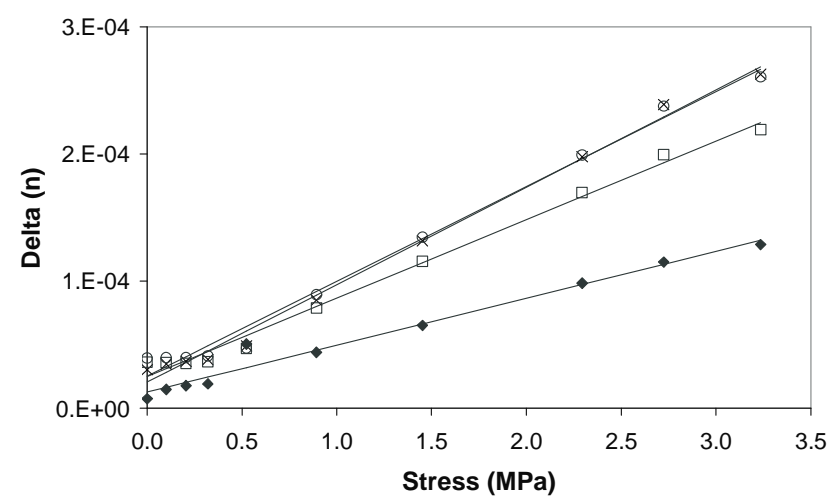

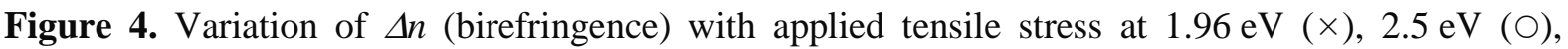
$3.5 \mathrm{eV}(\square)$ and $4.5 \mathrm{eV}(\bullet)$.

It is clear that large values of $\operatorname{Re}(\Delta \mathrm{R} / \mathrm{R})$ arise from birefringence provided that the $\mathrm{x} / \mathrm{y}$ Cartesian axes of the sample do not coincide with the optical fast/slow axes. The angle $\alpha$ is plotted as 
a function of strain in figure 5. It is seen to be a relatively small angle which increases rapidly with strain at low strains and exhibits an asymptotic behaviour at the higher strains.

In common with all polymers PDMS is made up of long chain molecules of repeat units. It has a (-Si-O-) backbone with pendent methyl groups on each $\mathrm{Si}$ atom. These pendant methyl groups minimise the interaction between adjacent chains thus reducing the barriers to rotation of the chains and allowing high flexibility. When the cross-linked networks are stretched under mechanical load there is a tendency for the linear chains to align with the direction of the load although the crosslink components will impede this alignment to a certain extent depending on the crosslink density. This behaviour is manifest in the engineering stress/strain curve which is shown in figure 5. Here there is a linear section extending to around 30\% strain beyond which the curve slope increases, corresponding to an increasing elastic modulus. This behaviour has previously been reported with Dow Corning Sylgard 184 [27] and GE Silicones RTV 615 PDMS [27]. Also shown in figure 5 is a quadratic fit to the stress/strain data, from which an effective Young's Modulus of $1.36 \mathrm{MPa}$ is obtained. It is noted that this is in fair agreement with 1.76 MPa for Sylgard 184 and 1.54 MPa for RTV 615. Therefore whilst the optical axes may coincide with the direction of the linear chain, these chains will not be fully aligned with the direction of the applied load and this would account for the fact that there is an angular difference between the mechanical frame, of which the load direction is one axis, and the optical frame. Indeed large changes in the magnitude of $\operatorname{Re}(\Delta R / R)$ with variations in the angle between the optical and mechanical axes of biaxial oriented polyethylene terephthalate [21] have been reported. Figure 5 compares the variation of $\alpha$ with strain on the one hand and stress with strain on the other. There appears to be correspondence between the rapid increase in $\alpha$ and the linear variation of strain whereas the asymptotic behaviour in $\alpha$ appears to be related to the departure from linearity in the stress-strain relationship. Both sets of results are consistent with there being two regimes resisting the alignment of the polymer chains. At the lower strains the elastically "soft" linear chains begin to align with the applied load. Above about $40 \%$ strain elastically "stiff" cross links begin to dominate and impede the alignment. 


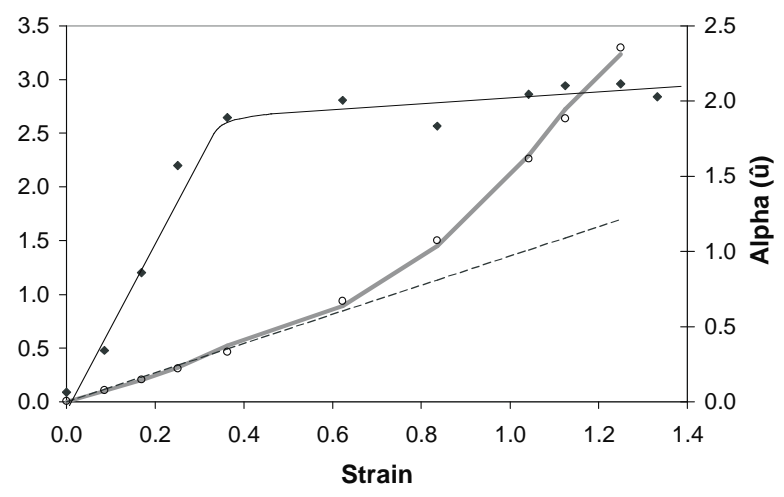

Figure 5. Stress-strain curve for the PDMS sample $(\bigcirc)$ with a quadratic fit to the data (grey line) and a linear fit to the first part of the data (dashed line) plotted on the primary $y$-axis and the alignment of the optical axes of the PDMS sample as a function of strain $(\bullet)$ is plotted on the secondary y-axis with a solid black line to aid the eye.

\section{Conclusion}

We have demonstrated that under mechanical loads the RA spectra of PDMS may be very large in magnitude. This is entirely due to the small stress birefringence exhibited by the material. When using PDMS as a substrate for studying any stress related anisotropy of surface deposited bio-molecules the bulk birefringence must be eliminated and we have demonstrated an effective method of achieving this.

Using expressions for $\Delta R / R$ derived from a Stokes-Mueller treatment of the optical system the RA results for the transmitted/reflected light over the spectral range $1.5 \mathrm{eV}$ to $4.7 \mathrm{eV}$ for all applied mechanical loads were simulated; each simulation was in good agreement with the measured values. The values of the difference in refractive indices between of the fast/slow axes so obtained enabled the stress-optical birefringence coefficient, $\mathrm{K}$, to be estimated at any photon energy in the above spectral range; the value of $\mathrm{K}$ agrees within a factor of two with that quoted in the literature at the $\mathrm{He}$ Ne laser energy. Finally the results are shown to be consistent with a network comprising elastically soft polymer chains cross linked with stiff bridges; this network also accounts for the measured stress/strain curve for our material which is similar to that published for PDMS from other sources. 


\section{Acknowledgements}

The authors would like to acknowledge financial support from the UK Engineering and Physical Sciences Research Council (EPSRC) for this work.

\section{References}

[1] Wipiff P-J, Majd H, Acharya C, Buscemi L, Meister J-J, Hinz B, 2009 Biomaterials 301781

[2] Lee J N, Jiang X, Ryan D and Whitesides G.M, 2004 Langmuir 2011691

[3] Shih T-K, Chen C-F, Ho J-R, and Chuang F-T, 2006 Microelectron. Eng. 832499

[4] Aspnes D E and Studna A A, 1985 Phys. Rev. Lett. 541956

[5] Kamiya I, Aspnes D E, Florez L T and Harbison J P, 1992 Phys. Rev. B 6415894

[6] Harbison J P, Aspnes D E, Studna A A, Florez L T and Kelly M K, 1988 Appl. Phys. Lett. 52 2046

[7] Weightman P, Martin D S, Cole R J and Farrell T, 2005 Rep. Prog. Phys. 681251

[8] Flores-Camacho J M, Weidlinger G, Saucedo-Zeni N, Sun L D, Hohage M and Zeppenfeld P, 2010 Appl. Phys. A 98499

[9] Richter W and McGilp J F, 1993 Phil. Trans. Roy. Soc. A 344453

[10] Rumberg J, 1996 Diploma Thesis, Technical University Berlin

[11] Richter W and Zettler J T, 1996 Appl. Surf. Sci. 100465

[12] Zettler J T, Pristovsek M, Trepk T, Shkrebtii A, Seimetz E, Zorn M and Richter W, 1998 Thin Solid Films 313537

[13] Mazine V, Borenstein Y, Cagnon L and Allogue P, 1999 Phys. Status Solidi A 175311

[14] Smith C I, Maunder A J, Lucas C A, Nichols R J and Weightman P, 2003 J Electrochem. Soc. 150 E233

[15] Smith C I, Dolan G J, Farrell T, Maunder A J, Fernig D G, Edwards C and Weightman P, 2004 J. Phys.: Condens. Matter 16 S4385

[16] McIntyre J D E and Aspnes D E, 1971 Surf. Sci. 24417

[17] Cole R J, Frederick B G and Weightman P, 1998 J. Vac. Sci. Technol. A 163088

[18] Mazine V and Borensztein Y, 2002 Phys. Rev. Lett. 88147403 
[19] Weightman P, Smith C I, Martin D S, Lucas C A, Nichols R J and Barrett S D, 2004 Phys Rev Lett. 92199707

[20] Schmidegg K, Sun L D and Zeppenfeld P, 2006 Appl. Phys. Lett. 89051906

[21] Haines S R, Beamson G, Williams R L and Weightman P, 2007 Surf. Interface Anal. 39942

[22] Aspnes D E, Harbison J P, Studna A A and Florez L T, 1988 J. Vac. Sci. Technol. A 61327

[23] Azzam R M A and Bashara N M 1997 Ellipsometry and Polarised Light (Amsterdam: Elsevier)

[24] van Krevelen D W and te Nijenhuis K 2009 Properties of Polymers: Their Correlation with Chemical Structure (Amsterdam: Elsevier) p 305

[25] Hoshino K and Shimoyama I, 2003 J Micromech. Microeng 13149

[26] Andriot M, DeGroot J V Jr and Meeks R, 2007 Silicones in Industrial Applications Inorganic Polymers ed R De Jaeger and M Gleria (New York: Nova Science) p 61

[27] Schneider F, Draheim J, Kamberger R and Wallrabe U, 2009, Sensor Actuat. A-Phys. 151, 95

[28] Wang B and Krause S, 1987, Macromolecules 20, 2201

[29] Armani B, Liu C and Aluru N, Proc IEEE Microelectro Mechanical Systems (MEMS) '99 Orlando Fl Jan 1999, 222 

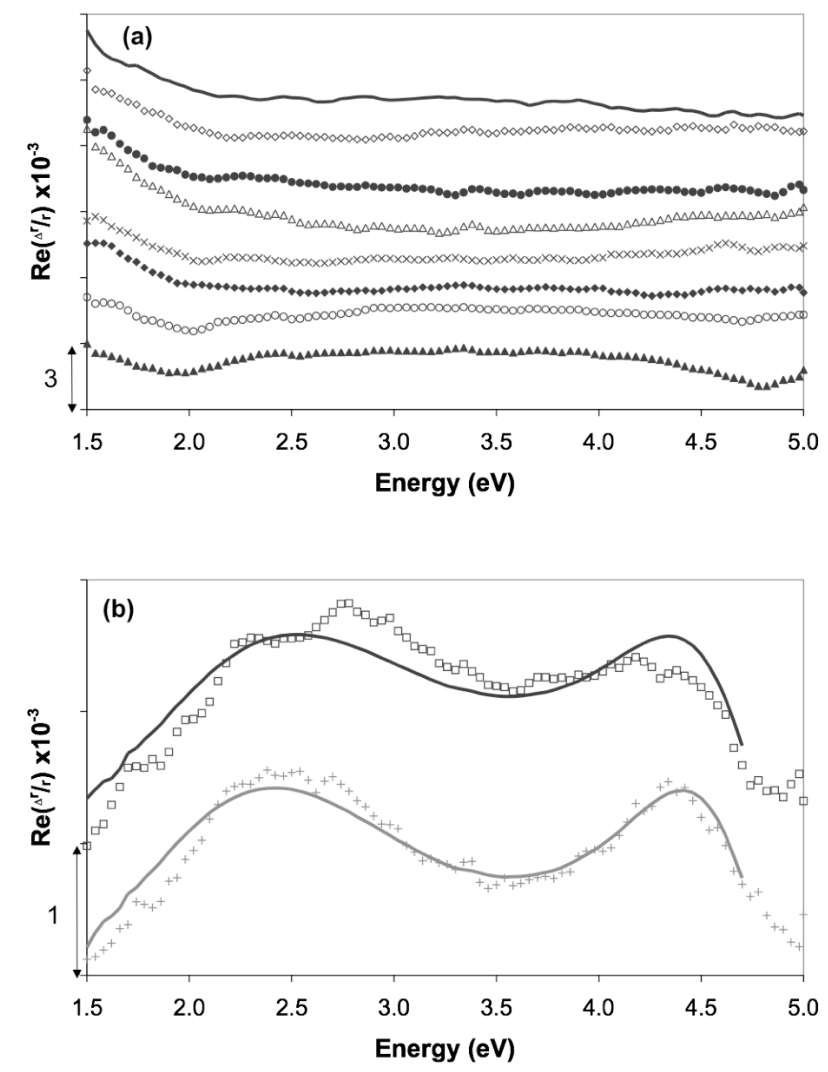


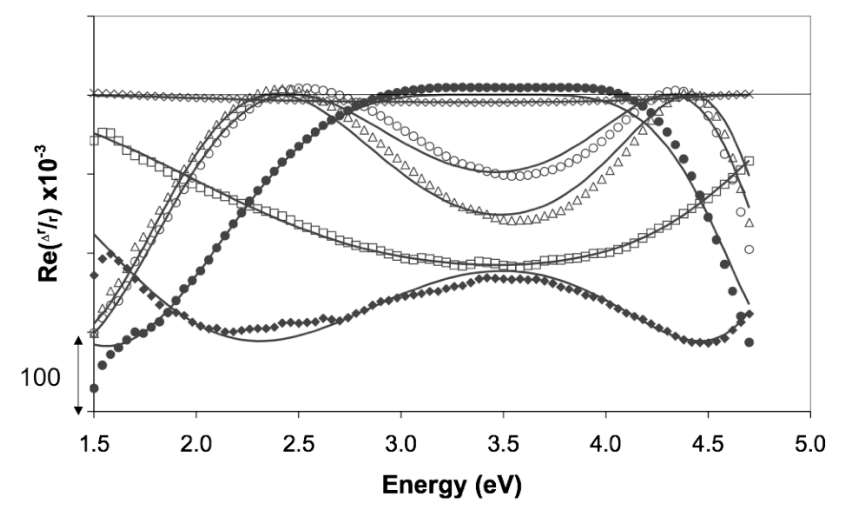




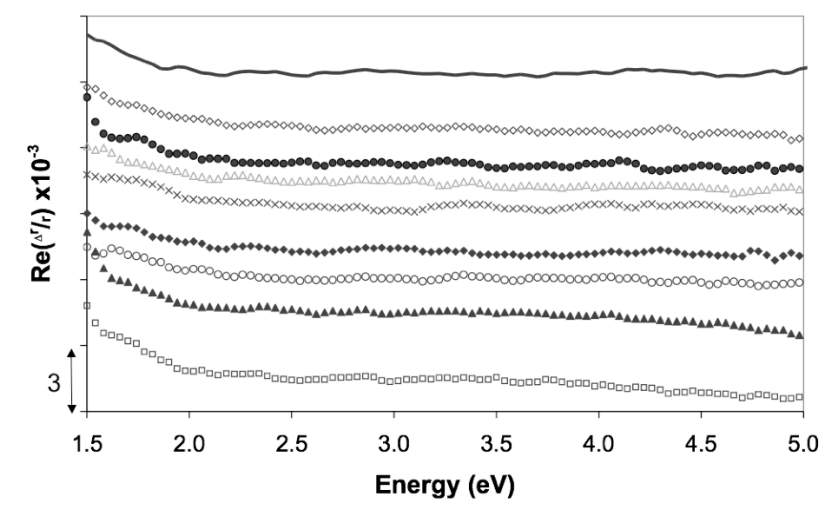




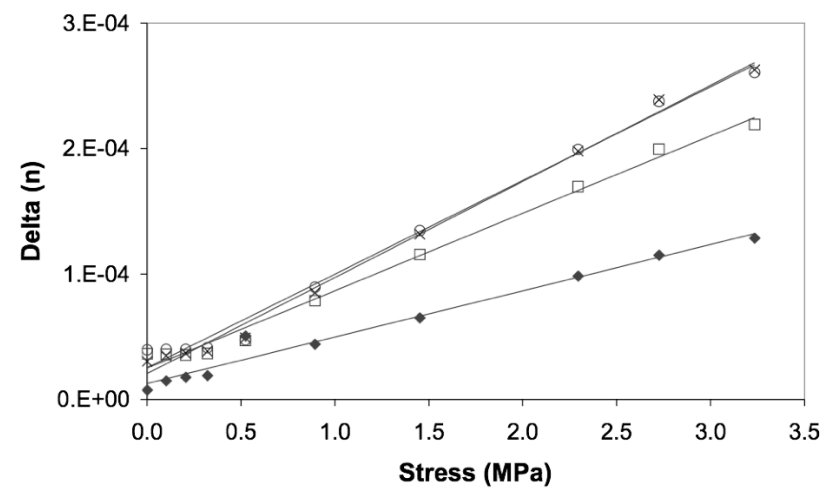




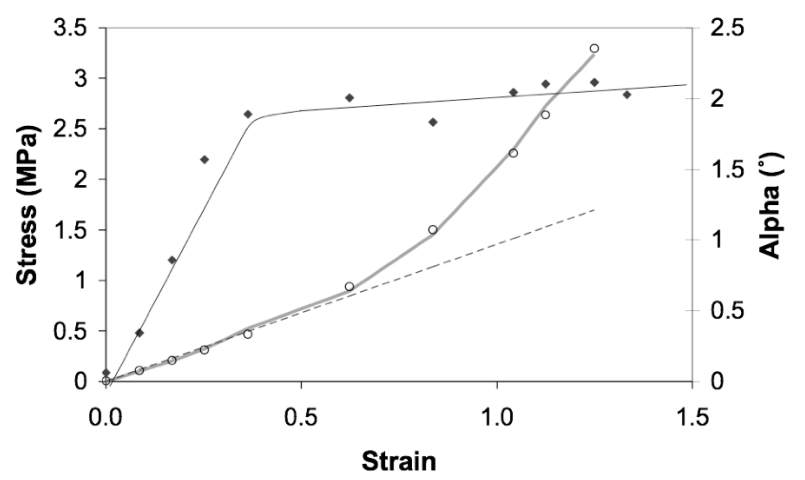

